\title{
DENSITY OF INFIMUM-STABLE CONVEX CONES
}

\author{
JOÃO B. PROLLA
}

(Communicated by Palle E. T. Jorgensen)

\begin{abstract}
Let $X$ be a compact Hausdorff space and let $A$ be a linear subspace of $C(X ; \mathbb{R})$ containing the constant functions, and separating points from probability measures. Then the inf-lattice generated by $A$ is uniformly dense in $C(X ; \mathbb{R})$. We show that this is a corollary of the Choquet-Deny Theorem, thus simplifying the proof and extending to the nonmetric case a result of McAfee and Reny.
\end{abstract}

Let $X$ be a compact Hausdorff space and let $C(X ; \mathbb{R})$ the space of all continuous real-valued functions be endowed with the sup-norm. Let $A$ be a linear subspace of $C(X ; \mathbb{R})$, containing the constant functions. Let

$$
\begin{aligned}
& A_{m}=\left\{\inf \left(f_{1}, \ldots, f_{n}\right) ; f_{i} \in A, 1 \leq i \leq n, n \in \mathbb{N}\right\}, \\
& A_{M}=\left\{\sup \left(f_{1}, \ldots, f_{n}\right) ; f_{i} \in A, 1 \leq i \leq n, n \in \mathbb{N}\right\} .
\end{aligned}
$$

Then $A_{m}$ (resp. $A_{M}$ ) is a convex conic inf-lattice (resp. sup-lattice), and McAfee and Reny [3] proved that $\overline{A_{m}}=\overline{A_{M}}=C(X ; \mathbb{R})$ if and only if $A$ separates points from probability measures, in the case $X$ is a metric space. Our aim is to give a simpler proof of the above result, which is valid even without the restriction of $X$ being a metric space. The proof is based on the classical Choquet-Deny Theorem (see Choquet-Deny [1] or Nachbin [4, §21]). We present the proof of an improved version of this result (see Theorem 1). Our proof follows closely the arguments of Nachbin [4].

Let us recall that a subset $S$ of $C(X ; \mathbb{R})$ is called an inf-lattice (resp. suplattice) or infimum-stable (resp. supremum-stable) subset if $f, g \in S$ implies $f \wedge g \in S$ (resp. $f \vee g \in S)$. Here $(f \wedge g)(x)=\inf (f(x), g(x))$ and $(f \vee g)(x)=$ $\sup (f(x), g(x))$, for all $x \in X$. On the other hand, $S \subset C(X ; \mathbb{R})$ is a convex cone if and only if $f, g \in S$ and $\lambda \geq 0$ imply $f+g \in S$ and $\lambda f \in S$.

Lemma 1. Let $S \subset C(X ; \mathbb{R})$ be an infimum-stable subset and, for each point $x \in X$, let $P_{x}=\{f \in C(X ; \mathbb{R}) ; f \geq 0, f(x)=0\}$. Then

$$
\bar{S}=\bigcap\left\{\overline{S-P_{x}} ; x \in X\right\} .
$$

Proof. For each $x \in X$, we have $0 \in P_{x}$. Hence $\bar{S} \subset \overline{S-P_{x}}$, for each $x \in X$. Conversely, assume that $f \in \overline{S-P_{x}}$, for each $x \in X$. Let $\varepsilon>0$ be given. For each $x \in X$, there are $g_{x} \in S$ and $h_{x} \in P_{x}$ such that $\left\|g_{x}-h_{x}-f\right\|<$

Received by the editors August 20, 1992.

1991 Mathematics Subject Classification. Primary 46B28. 
$\varepsilon / 2$. Let $V_{x}=\left\{t \in X ; h_{x}(t)<\varepsilon / 2\right\}$. Then $V_{x}$ is open and contains $x$. By compactness, there are $x_{1}, \ldots, x_{n} \in X$ such that $X=V_{x_{1}} \cup \cdots \cup V_{x_{n}}$. Let $g=\inf \left\{g_{x_{1}}, \ldots, g_{x_{n}}\right\}$. Then $g \in S$. Let $t \in X$. Then, for each $j=1, \ldots, n$, we have

$$
g_{x_{j}}(t) \geq g_{x_{j}}(t)-h_{x_{j}}(t)>f(t)-\varepsilon / 2 .
$$

Hence $g(t)>f(t)-\varepsilon$. On the other hand, there is some index $i$ such that $t \in V_{x_{i}}$, and then $h_{x_{i}}(t)<\varepsilon / 2$ and $g(t) \leq g_{x_{i}}(t)$ imply $g(t)-\varepsilon / 2<g_{x_{i}}(t)-$ $h_{x_{i}}(t)<f(t)+\varepsilon / 2$. Hence $g(t)<f(t)+\varepsilon$. Therefore $\|f-g\|<\varepsilon$ and $f \in \bar{S}$.

Lemma 2. Let $\varphi$ be a nonzero continuous linear form on $C(X ; \mathbb{R})$ and let $x \in X$. If $\varphi$ is positive on $P_{x}=\{f \in C(X ; \mathbb{R}) ; f \geq 0, f(x)=0\}$, there is $r \in \mathbb{R}$ such that $r<0$ and $\varphi \geq r \delta_{x}$.

Proof (Nachbin [4, $§ 21]$ ). Assume that $\varphi(f) \geq 0$ for all $f \geq 0$ such that $f(x)=0$. Let

$$
B=\{f \in C(X ; \mathbb{R}) ; f \geq 0, f(x)=1\} .
$$

For any $f \in B$, notice that $g=f-\inf (1, f)$ belongs to $P_{x}$. Hence $\varphi(g) \geq$ 0 and so $\varphi(f) \geq \varphi(\inf (1, f))$. Now $0 \leq \inf (1, f) \leq 1$ and therefore $|\varphi(\inf (1, f))| \leq\|\varphi\|$. Hence $\varphi(f) \geq-\|\varphi\|$, for all $f \in B$. Let $r=-\|\varphi\|$.

Let $f \geq 0$ be given in $C(X ; \mathbb{R})$. If $f(x)>0$, then $\varphi(f / f(x)) \geq r$ and so $\varphi(f) \geq r f(x)$. If $f(x)=0$, then $f \in P_{x}$ and so $\varphi(f) \geq 0=r f(x)$. Hence, $\varphi \geq r \delta_{x}$.

If $S \subset C(X ; \mathbb{R})$ is an infimum-stable convex cone, let $\Gamma(S)$ be the set of all pairs $(x, \varphi)$, where $x$ belongs to $X$ and $\varphi$ is a positive linear form on $C(X ; \mathbb{R})$, such that

$$
\varphi(g) \leq g(x), \quad \text { for all } g \in S \text {. }
$$

Let $\widehat{K}(S)$ be the set of all functions $f \in C(X ; \mathbb{R})$ such that $\varphi(f) \leq f(x)$, for all $(x, \varphi) \in \Gamma(S)$. With this notation, the following improved version of the Choquet-Deny Theorem is true.

Theorem 1. Let $S \subset C(X ; \mathbb{R})$ be an infimum-stable convex cone. Then $\bar{S}=$ $\widehat{K}(S)$.

Proof. It is easy to see that $\widehat{K}(S)$ is a closed subset containing $S$. Hence $\bar{S} \subset \widehat{K}(S)$.

Conversely, let $f \in C(X ; \mathbb{R})$ be such that $f \notin \bar{S}$. By Lemma 1 , there exists some $x \in X$ such that $f \notin \overline{S-P_{x}}$. Since $S-P_{x}$ is convex, by the HahnBanach Theorem there is a nonzero continuous linear form $\psi$ on $C(X ; \mathbb{R})$ and $c \in \mathbb{R}$ such that $\psi(g-h) \leq c<\psi(f)$ for all $g \in S$ and $h \in P_{x}$. Since $0 \in S$, we have $\psi(-\lambda h) \leq c$, for all $\lambda>0$. Dividing by $\lambda$ and letting $\lambda \rightarrow \infty$ we get $\psi(h) \geq 0$, for all $h \in P_{x}$. By Lemma 2, there is $r \in \mathbb{R}$ such that $r<0$ and $\psi \geq r \delta_{x}$. Then $\varphi=\delta_{x}-r^{-1} \psi$ is positive. Since $0 \in P_{x}$, we have $\psi(g) \leq c<\psi(f)$, for every $g \in S$. Hence

$$
\varphi(g)-g(x)=-r^{-1} \psi(g) \leq-r^{-1} c<-r^{-1} \psi(f)=\varphi(f)=f(x) .
$$

Since $\lambda g \in S$, for all $\lambda>0$, we have $\varphi(\lambda g)-\lambda g(x) \leq-r^{-1} c$. Dividing by $\lambda$ and letting $\lambda \rightarrow \infty$, we get $\varphi(g)-g(x) \leq 0$, for all $g \in S$. On the other hand, 
$0 \in S$ implies $0=\varphi(0)-0 \leq-r^{-1} c<\varphi(f)-f(x)$, and so $f(x)<\varphi(f)$. Hence $f \notin \widehat{K}(S)$.

Corollary 1. Let $S \subset C(X ; \mathbb{R})$ be an infimum-stable convex cone. Then $S$ is uniformly dense if and only if the following is true: for every $f \in C(X ; \mathbb{R})$, one has $\varphi(f) \leq f(x)$ whenever $(x, \varphi) \in \Gamma(S)$.

Let us recall that $A$ is said to separate points from probability measures if for any probability measure $\mu$ on $X$, and any $x \in X$, such that $\mu(g)=g(x)$, for all $g \in A$, then necessarily $\mu=\delta_{x}$, the Dirac measure at $x$.

Theorem 2. Let $A$ be a linear subspace of $C(X ; \mathbb{R})$ such that $1 \in A$. Then $A_{m}$ is uniformly dense if, and only if, $A$ separates points from probability measures.

Proof. Let $S=A_{m}$. Then $S$ is an infimum-stable convex cone.

$(\Rightarrow)$ Assume $S$ is dense, and let $x \in X$ and $\mu$ a probability measure on $X$ be given such that $f(x)=\mu(f)$, for all $f \in A$. Then $g(x) \geq \mu(g)$, for all $g \in S$. Let $h \in C(X ; \mathbb{R})$. By Theorem $1, h(x) \geq \mu(h)$ and $-h(x) \geq \mu(-h)$. Hence $h(x)=\mu(h)$. This shows that $A$ separates points from probability measures.

$(\Leftarrow)$ Conversely, assume that the subspace $A$ separates points from probability measures. Let $(x, \varphi) \in \Gamma(S)$. For each $g \in A$, both $g$ and $-g$ belong to $\widehat{K}(S)$, since $A \subset S$, and therefore $g(x)=\varphi(g)$, for all $g \in A$. The fact that $1 \in A$, implies $1=\mathbf{1}(x)=\varphi(\mathbf{1})$. Therefore $\varphi$ is a probability measure on $X$, and then $f(x)=\varphi(f)$, for all $f \in C(X ; \mathbb{R})$. Hence $\widehat{K}(S)=C(X ; \mathbb{R})$ and by Corollary $1, S$ is uniformly dense.

Corollary 2. Let $A$ be a linear subspace of $C(X ; \mathbb{R})$ such that $1 \in A$. Then $\overline{A_{m}}=\overline{A_{M}}=C(X ; \mathbb{R})$ if, and only if, $A$ separates points from probability measures.

Proof. This follows from Theorem 2 and the fact that $A_{M}=-(-A)_{m}=$ $-A_{m}$.

Remark 1. Let us recall the notion of the Choquet boundary of a linear subspace $A$ of $C(X ; \mathbb{R})$, denoted by $\partial_{A} X$. By definition,

$$
\partial_{A} X=\left\{x \in X ; A(x)=\left\{\delta_{x}\right\}\right\}
$$

where $A(x)=\{\mu \in \Delta ; \mu(g)=g(x)$, for all $g \in A\}$, and $\Delta$ is the set of all probability measures on $X$.

Theorem 3. Let $A$ be a linear subspace of $C(X ; \mathbb{R})$ such that $1 \in A$. The following are equivalent:

(1) $\overline{A_{m}}=\overline{A_{M}}=C(X ; \mathbb{R})$.

(2) A separates points from probability measures.

(3) $\partial_{A} X=X$.

Proof. By Corollary 2, (1) $\Leftrightarrow(2)$.

(2) $\Rightarrow(3)$ Let $x \in X$ be given. Let $\mu \in A(x)$. Then $\mu(g)=g(x)$, for all $g \in A$. Since $A$ separates points from probability measures, this implies that $\mu(f)=f(x)$, for all $f \in C(X ; \mathbb{R})$, i.e., $\mu=\delta_{x}$. Hence $x \in \partial_{A} X$, and so $X=\partial_{A} X$.

(3) $\Rightarrow$ (2) Let $f \in C(X ; \mathbb{R})$ be given. Let $x \in X$ and $\varphi \in \Delta$ be such that $\varphi(g)=g(x)$, for all $g \in A$. Then $\varphi \in A(x)$. Since $x \in \partial_{A} X$, it follows 
that $\varphi=\delta_{x}$. Hence $\varphi(f)=f(x)$, and $A$ separates points from probability measures.

Remark 2. The equivalence (1) $\Leftrightarrow$ (3) is due to Flösser, Irmisch, and Roth [2]. (See Example 4.2 of [2].) Since the equivalence $(2) \Leftrightarrow(3)$ is almost obvious, the paper [2] gives an alternative proof of the equivalence $(1) \Leftrightarrow(2)$.

\section{REFERENCES}

1. G. Choquet and J. Deny, Ensembles semi-réticulés et ensembles réticulés de fonctions continues, J. Math. Pures Appl. 36 (1957), 179-189.

2. H. O. Flösser, R. Irmisch, and W. Roth, Infimum-stable convex cones and approximation, Proc. London Math. Soc. (3) 42 (1981), 104-120.

3. R. P. McAfee and P. Reny, A Stone-Weierstrass theorem without closure under suprema, Proc. Amer. Math. Soc. 114 (1992), 61-67.

4. L. Nachbin, Elements of approximation theory, Van Nostrand, Princeton, NJ, 1967; reprinted by Krieger, Huntington, NY, 1976.

Department of Mathematics, IMECC, UNICAMP, Caixa Postal 6065, Campinas, Brazil E-mail address: prolla@ime.unicamp.br 